\title{
Intensity-based elastic registration incorporating anisotropic landmark errors and rotational information
}

\section{Journal Article}

Author(s):

Šerifović-Trbalić, Amira; Demirović, Damir; Prljača, Nasr; Székely, Gábor; Cattin, Philippe C.

Publication date:

2009

Permanent link:

https://doi.org/10.3929/ethz-b-000021891

Rights / license:

In Copyright - Non-Commercial Use Permitted

Originally published in:

International Journal of Computer Assisted Radiology and Surgery 4(5), https://doi.org/10.1007/s11548-009-0358-2 


\title{
Intensity-based elastic registration incorporating anisotropic landmark errors and rotational information
}

\author{
A. Šerifović-Trbalić · D. Demirović • N. Prljača • \\ G. Szekely • Philippe C. Cattin
}

Received: 14 January 2009 / Accepted: 4 May 2009 / Published online: 4 June 2009

(c) CARS 2009

\begin{abstract}
Purpose Thin-plate splines (TPS) represent an effective tool for estimating the deformation that warps one set of landmarks to another based on the physical equivalent of thin metal sheets. In the original formulation, data used to estimate the deformation field are restricted to landmark locations only and thus does not allow to incorporate information about the rotation of the image around the landmark. It furthermore assumes that landmark positions are known exactly which is not the case in real world applications. These localization inaccuracies are propagated to the entire deformation field as each landmark has a global influence. We propose to use a TPS approximation method that incorporates anisotropic landmark errors and rotational information and integrate it into a hierarchical elastic registration framework (HERA). The improvement of the registration performance has been evaluated.

Methods The proposed TPS approximation scheme integrates anisotropic landmark errors with rotational information of the landmarks. The anisotropic landmark errors are represented by their covariance matrices estimated directly
\end{abstract}

This work has been supported by the SCOPES program of the Swiss National Science Foundation (http://www.snf.ch).

A. Šerifović-Trbalić · D. Demirović · N. Prljača

Faculty of Electrical Engineering, University of Tuzla,

75000 Tuzla, Bosnia and Herzegovina

e-mail: amira.serifovic@untz.ba

G. Szekely

Computer Vision Laboratory, ETH Zurich,

8092 Zurich, Switzerland

P. C. Cattin $(\varangle)$

Medical Image Analysis Center, University of Basel,

4031 Basel, Switzerland

e-mail: philippe.cattin@unibas.ch from the image data as a minimal stochastic localization error, i.e. the Cramér-Rao bound. The rotational attribute of each landmark is characterized by an additional angular landmark, thus doubling the number of landmarks in the TPS model. This allows the TPS approximation to better cope up with local deformations.

Results We integrated the proposed TPS approach into the HERA registration framework and applied it to register 161 image pairs from a digital mammogram database. Experiments showed that the mean squared error using the proposed TPS approximation was superior to pure TPS interpolation. On artificially deformed breast images HERA, with the proposed TPS approximation, performed significantly better than the state-of-the-art registration method presented by Rueckert.

Conclusion The TPS approximation approach proposed in this publication allows to incorporate anisotropic landmark errors as well as rotational information. The integration of the method into an intensity-based hierarchical non-rigid registration framework is straightforward and improved the registration quality significantly.

Keywords Thin plate splines - Image registration Approximation

\section{Introduction}

Medical image registration is a difficult and ill-posed problem. Numerous methods and tools have been proposed to address this task, see $[8,16]$ and the references therein. One key element common to all these methods is the need to interpolate a dense deformation field from sparse known correspondences. For parametric models, such as rigid or affine transformations, this interpolation step is trivial but 
they lack the necessary degrees of freedom $(d f)$ to accurately compensate for local deformations. Schaeffer et al. proposed in [15] a locally rigid or affine Moving Least Squares approach that is more flexible and still computationally efficient. A yet different polyrigid transformation approach was proposed by Arsigny et al. [2]. Other types of transformations, such as B-splines [14], thin-plate splines (TPS) [4] or continuum mechanics based approaches (like finite elements) offer more general deformation models that can have an arbitrary number of $d f$.

Thin-plate splines are often used to interpolate a dense deformation field in landmark and intensity-based image registration. TPS were first proposed in 1976 by Duchon [7] for surface fitting and then applied to landmark-based registration of images by Bookstein et al. [4] in 1989. TPS represent an effective tool for estimating the deformation that warps one set of landmarks to another based on the physical equivalent of thin metal sheets. Data used to estimate the deformation field are restricted to landmark locations only in the original formulation and thus do not allow to incorporate information about the rotation of the image around the landmark. It furthermore assumes that landmark positions are known exactly which is not the case in real world applications. These localization inaccuracies are propagated to the entire deformation field as each landmark has a global influence. To model complex and localized deformations many landmark have to be used at the expense of high computational cost.

Various methods have been described in the literature to address these problems. Rohr et al. [11,13] proposed a regularized interpolation scheme, which provides a smoothing function that approximates rather than strictly interpolate. A different approach was proposed by Lewis et al. [9] who tackles the inaccuracies by replacing the ambiguous landmarks along a contour.

An extension that further improves TPS' capability to cope up with local deformations is the integration of the derivatives such as tangent and curvature information. Bookstein and Green [5] describe a TPS-based approximation method that incorporates the tangent direction, which is used to characterize the local orientation of the contours at the landmarks. The orientations are characterized through additional points placed close to these landmarks. The limitation of their approach is that changes in the landmarks and their derivatives in one part of the image can have an appreciable effect on the deformation in distant locations. The Kriging method presented by Mardia et al. [10] also uses the derivative. Their interpolation scheme yields an exact orientation, but it requires that the corresponding vectors have to be of unit length. It should be noted that both Bookstein's and Mardia's approach are interpolation schemes, although a generalization to approximation is possible. A yet different approach was proposed by Rohr et al. [12], which includes the orientation constraints as scalar products of the normalized orientation vectors. His landmark-based registration approach, however, requires user intervention to select the landmarks based on localization uncertainty and to estimate their orientation attributes. Their approach shows a substantial improvement, but landmarks should be well distributed in order to achieve good registration results.

In this work, we present an extended TPS model that incorporates anisotropic landmark errors as well as rotational information. We furthermore show that its integration into a fully automatic hierarchical elastic image registration method [1] is possible and significantly improves the registration result.

\section{Method}

The proposed TPS model is integrated into the elastic hierarchical image registration framework (HERA) proposed in [1], which decomposes a non-rigid registration problem into numerous local rigid transformations, see Fig. 1a. The procedure entails multiple hierarchical levels. At each level, the floating image, pre-registered with the reference image at the higher level, is partitioned into progressively smaller
Fig. 1 a Hierarchical registration scheme, b angle points

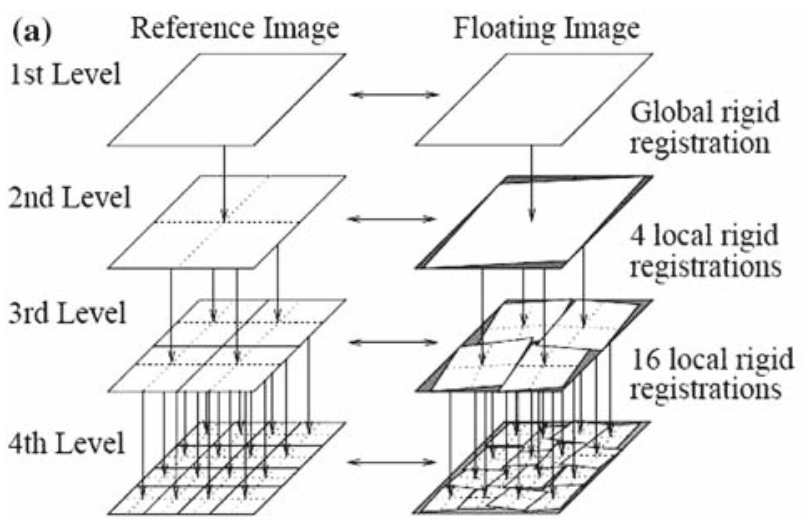

(b)

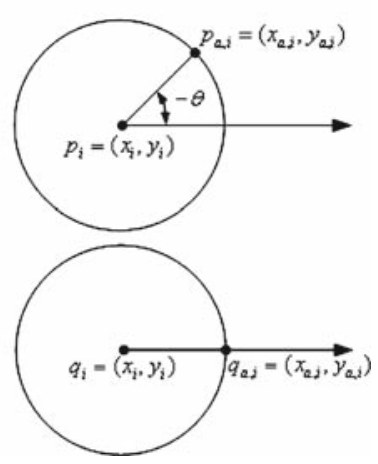


sub-images. Each floating sub-image is then rigidly registered with the corresponding part of the reference image by maximizing mutual information. After each level, outlier sub-images are detected using consistency tests. The final dense deformation field is then obtained with the proposed TPS interpolation scheme. The set of landmarks for the TPS is formed by the centers and rotation of all terminating sub-images. Hence, the landmarks and the corresponding rotation angles are automatically obtained from the hierarchical procedure.

Our TPS approximation scheme combines the rotation information of the image around landmarks using a variant of the method proposed by Chen [6] with the anisotropic landmark localization errors from Rohr [11]. This approach is generally more accurate and allows, in contrast to [12], a fully automatic implementation.

Let the location of the $n$ floating landmarks, i.e. the centers of the sub-images, be denoted by $p_{i}=\left(x_{i}, y_{i}\right), i=1, \ldots, n$ and the rotation angle of each sub-image as $\theta_{i}$. The unit circle around each landmark $p_{i}$ and its corresponding angular landmark $p_{a, i}=\left(x_{a, i}, y_{a, i}\right)$, see Fig. $1 \mathrm{~b}$, can thus be obtained by

$x_{a, i}=x_{i}+\cos \left(-\theta_{i}\right), \quad y_{a, i}=y_{i}+\sin \left(-\theta_{i}\right)$.

Each of these newly created angular landmarks needs a corresponding landmark in the reference image. These additional reference landmarks $q_{a, i}=\left(x_{a, i}, y_{a, i}\right)$ can be straightforwardly determined as

$x_{a, i}=x_{i}+1, \quad y_{a, i}=y_{i}$.

The rotational attribute of each landmark is thus converted into an additional angular landmark doubling the number of landmarks in the TPS model.

The incorporation of the anisotropic landmark errors in the TPS model was taken from [13] and is briefly summarized in the following. The anisotropic landmark localization errors can be represented by their covariance matrices $\Sigma_{i}$ estimated directly from the image data. In this case, the covariance matrices represent the minimal stochastic localization error, i.e. the Cramér-Rao bound, which can be written as

$\Sigma_{i}=\frac{\sigma_{n}^{2}}{m} C_{g}^{-1}$

where $\sigma_{n}^{2}$ denotes the variance of additive white Gaussian image noise, $m$ the number of pixels in a local neighborhood, and $C_{g}$ is the averaged dyadic product of the image gradient with $g(\mathbf{x})$ being the image function. Let the doubled landmark sets be denoted by $\mathbf{p}_{\text {new }}=\left\{\mathbf{p}, \mathbf{p}_{a}\right\}$ and $\mathbf{q}_{\text {new }}=\left\{\mathbf{q}, \mathbf{q}_{a}\right\}$. By including the information about the landmark localization error into minimization, the base functional of the TPS
[12] can be re-written as

$$
\begin{aligned}
J_{\lambda}(\mathbf{u})= & \frac{1}{n} \sum\left(\mathbf{q}_{\text {new }, i}-\mathbf{u}\left(\mathbf{p}_{\text {new }, \mathrm{i}}\right)\right)^{T} \Sigma_{i}^{-1}\left(\mathbf{q}_{\text {new }, i}\right. \\
& \left.-\mathbf{u}\left(\mathbf{p}_{\text {new }, i}\right)\right)+\lambda J_{m}^{d}(\mathbf{u}) .
\end{aligned}
$$

The first term measures the distance between the two landmark sets weighted by the covariance matrices $\Sigma_{i}$, whereas the second term represents the smoothness of the transformation. The parameter $\lambda$ weights between these two terms. It should be noted that the $\Sigma_{i}$ represent the localization errors of corresponding landmark pairs, therefore the covariance matrices of corresponding landmarks have to be combined. Similar to Rohr et al. [13], we assume that the two covariance matrices only slightly depend on the nonlinear part of the transformation and that the images have a similar orientation, scale and shear. With these assumptions the two covariance matrices can be simply summed to form $\Sigma_{i}$.

This functional (4) has an analytic solution which can be written as

$$
\begin{aligned}
u_{k}(\mathbf{x})= & \sum_{v=1}^{M} a_{k, v} \phi_{v}(\mathbf{x}) \\
& +\sum_{i=1}^{n} w_{k, i} U\left(\mathbf{x}, \mathbf{p}_{\text {new }, i}\right), \quad k=1, \ldots, d
\end{aligned}
$$

with monomials $\phi$ up to the order $m-1$ and a suitable radial basis function $U\left(\mathbf{x}, \mathbf{p}_{\text {new }, i}\right)=\left\|\mathbf{x}-\mathbf{p}_{\text {new }, i}\right\|^{2} \log \left\|\mathbf{x}-\mathbf{p}_{\text {new }, i}\right\|^{2}$. Here $m$ represents the order of the involved derivatives of $u, d$ is the image dimension and $M=d+m-1$ represents the dimension of the nullspace of the functional (4). The unknown coefficients $\mathbf{a}=\left(\mathbf{a}_{1}^{T}, \ldots, \mathbf{a}_{M}^{T}\right)^{T}$ and $\mathbf{w}=$ $\left(\mathbf{w}_{1}^{T}, \ldots, \mathbf{w}_{M}^{T}\right)^{T}$ can be calculated from the following system of linear equations

$\left(\mathbf{K}+n \lambda \mathbf{W}^{-1}\right) \mathbf{w}+\mathbf{P a}=\mathbf{v}$,

$\mathbf{P}^{T} \mathbf{w}=\mathbf{0}$

where $\mathbf{W}^{-1}=\operatorname{diag}\left\{\Sigma_{1}, \ldots, \Sigma_{n}\right\}, \mathbf{K}=\left(U\left(\mathbf{p}_{\text {new }, i}, \mathbf{p}_{\text {new }, j}\right)\right.$ $\left.\mathbf{I}_{d}\right), \mathbf{P}=\left(\phi_{j}\left(\mathbf{p}_{\text {new }, i}\right) \mathbf{I}_{d}\right)$ and $\mathbf{I}_{d}$ is the $d \times d$ identity matrix. The vector $\mathbf{v}$ is the column vector of the $\mathbf{q}_{\text {new, } i}$ coordinates.

All subsequent statistical analyses were performed using the software package $\mathrm{R}$ (release 2.7.1 for Linux, http://www.r-project.org). The non-Gaussian distributed MSE results were compared with a Wilcoxon signed and paired rank test. A $p$ value $<0.05$ was considered statistically significant for all tests.

\section{Results and discussion}

We integrated the proposed TPS approximation in the elastic registration scheme [1] and applied it to register pairs of images of the same patient acquired during follow-up 
investigations, provided by the MIAS $^{1}$ digital mammogram database. The MIAS database consists of breast images of 161 patients obtained from X-ray film. The altogether 322 images have been classified into three groups, namely of normal breasts (208 images) and those showing benign (63 images) and malignant (51 images) lesions. Experts additionally labeled the images with four kinds of abnormities (architectural distortions, stellate lesions, circumscribed mass and calcifications).

To evaluate the performance of the proposed TPS approximation scheme three independent experiments were conducted. For all these experiments the following parameter settings were used: (a) the localization uncertainty $C_{g}$ was estimated using $\sigma_{n}^{2}=25$ in a $5 \times 5$ window; (b) the regularization parameter $\lambda$ was set to 0.01 ; (c) the $2 \mathrm{D}$ histogram was generated using 256 bins $(256 \times 256$ normalized gray values).

First, we demonstrate the benefit of incorporating rotation information at landmarks. The mean squared error (MSE) was used to quantify the registration quality of (1) TPS with anisotropic errors, (2) TPS with rotational information, and (3) the proposed TPS with anisotropic errors and rotational attributes all in relation to pure TPS interpolation. The evaluation was performed separately on the 161 image pairs. Significant non-rigid deformations can be observed in a group of 32 images with architectural distortions and/or asymmetries. Table 1 summarizes the MSE improvement and Fig. 2 shows an example registration as well as the difference image.

The results are very encouraging and the proposed approach significantly improved $p<0.05\left(p=10^{-16}\right)$ all registrations with an average improvement of the MSE as compared to pure TPS interpolation with $8.7 \pm 9.9 \%$ for symmetric and $8.8 \pm 13.2 \%$ for asymmetric mammograms. The table also clearly shows that the combination of the rotational attributes and anisotropic landmark errors performs best and thus justifies its higher computational costs. The average increase factor in run time in relation to pure TPS interpolation is shown in the right most column.

In the second experiment, we compare the hierarchical non-rigid registration [1] using our TPS approximation scheme with the state-of-the-art non-rigid registration approach proposed by Rueckert et al. [14]. Rueckert's method models the global motion with an affine transformation, while the local deformations are described by a free-form deformation (FFD) based on B-Splines. As the similarity criterion, which measures the degree of alignment between two images, they use mutual information.

For the 161 analyzed image pairs from the MIAS database, our hierarchical approach performed significantly $\left(P \approx 10^{-9}\right.$ ) better, by providing superior results for 128

\footnotetext{
${ }^{1} \mathrm{http} / / /$ peipa.essex.ac.uk/ipa/pix/mias.
}

Table 1 Average MSE improvement in relation to pure TPS interpolation

\begin{tabular}{llll}
\hline $\begin{array}{l}\text { Spatial } \\
\text { transformation }\end{array}$ & $\begin{array}{l}\text { MSE } \\
\text { normal [\%] }\end{array}$ & $\begin{array}{l}\text { MSE } \\
\text { asymm. [\%] }\end{array}$ & Run-time \\
\hline $\begin{array}{l}\text { TPS with } \\
\text { anisotropic errors }\end{array}$ & $0.8 \pm 17.7$ & $6.9 \pm 15.9$ & $1.1 \pm 0.1$ \\
$\begin{array}{l}\text { TPS with } \\
\text { rotation attributes }\end{array}$ & $1.1 \pm 5.9$ & $1.3 \pm 4.4$ & $1.7 \pm 0.2$ \\
$\begin{array}{l}\text { TPS with rotational } \\
\text { and anisotropic errors }\end{array}$ & $8.7 \pm 9.9$ & $8.8 \pm 13.2$ & $2.2 \pm 0.3$ \\
\hline
\end{tabular}

The right most column shows the average increase factor in run-time in relation to pure TPS interpolation

cases, while in six cases both approaches performed poorly. These cases can be attributed to large architectural distortions ( 4 cases) or lesions ( 2 cases), but there was no clear misalignment between the images. The proposed approach achieved an average MSE decrease in percentage of $9.7 \pm 37.0 \%$ compared to Rueckert's method. Table 2 gives the average values of the MSE, mutual information (MI) and correlation coefficient (CC) for Rueckert's and our approach calculated from the reference and the registered images.

The number of landmarks used to model the final deformation field in our registration method depends on the structural content of the image. To make the two methods comparable we tuned Rueckert's approach such that the average landmarks of both methods were approximately equal. In particular, we used on average 625 landmarks in Rueckert's and 595 landmarks in our registration approach.

In a third experiment, we artificially deformed ten images of different patients from the MIAS database. To obtain the deformation field, eight landmarks were chosen along the contour of the breast in the original image. Then each landmark was randomly translated and rotated (Fig. 3), where the range of translation and rotation was limited to 5-20 pixels and $0^{\circ}-30^{\circ}$. Finally, the known deformed reference image was obtained using TPS interpolation. To avoid correlated noise, the resulting images were modulated with white noise with zero mean and variance equal to $1 \%$ of the maximum amplitude. Because we artificially deformed the image, the exact deformation vector of each pixel is known. Hence, we can determine the average error per pixel for both registration approaches. With a mean error and standard deviation of $5.86 \pm 6.67$ pixels (min 0.05, $\max 19.77$ ) for Rueckert's approach, our method was significantly better $(P=0.027)$ having a mean and standard deviation of $0.88 \pm 0.86$ pixels (min 0.02, max 2.58). The black regions in the registered images (air) were ignored when calculating the above errors, as these regions can not be properly registered anyway. Figure 4 shows an example registration for both methods. The average error per pixel of registered breast tissue by 
Fig. 2 a The reference image, b asymmetric floating image, $\mathbf{c}$ the registered image using the proposed TPS interpolation, $\mathbf{d}$ difference between the reference and registered image
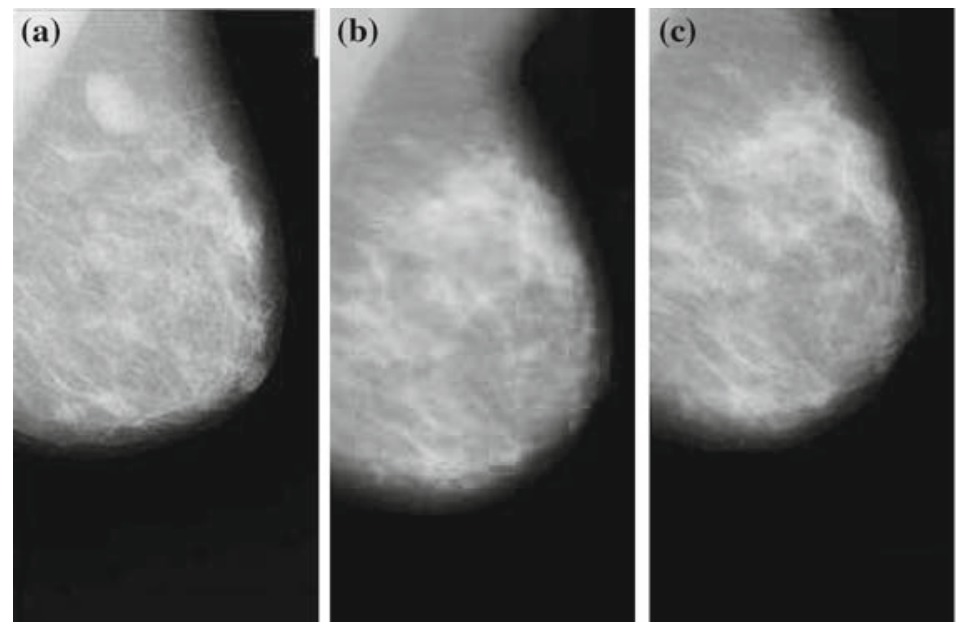

(d)

by Berthaud et al. [3]. Although Rueckert's approach is also based on a B-Spline transformation model, it did not perform significantly better $(P=0.064)$ than our TPS-based approach. The average error per pixel for Rueckert's method was $1.34 \pm 1.17$ pixels ( $\min 0.03$, $\max 3.65$ ), while our procedure resulted in $1.83 \pm 1.43$ pixels ( $\min 0.04$, $\max 4.02$ ).

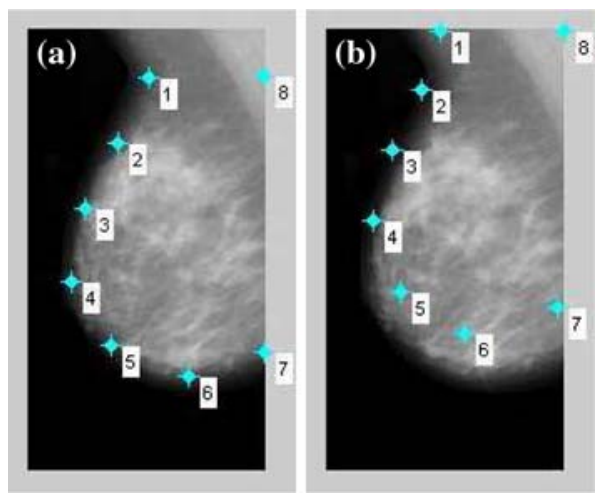

Fig. 3 a The initial position of landmarks, b position of the landmarks after translation and rotation
Rueckert and our approach for this example was 8.40 and 0.60 pixels, respectively.

To avoid the bias when registering images using TPS that were artificially deformed with TPS we performed the same test using B-Spline deformations. For this, the same ten images were artificially deformed with B-Splines to get the deformation field. First the uniform grid, that is needed for B-Spline interpolation, of size $8 \times 8$ was generated. The eight grid points closest to the landmarks used during TPSbased deformation were translated and rotated using the same parameters as for the TPS landmarks. The remaining grid points were left at their initial position. Rotations of the B-Splines grid were implemented using the method proposed
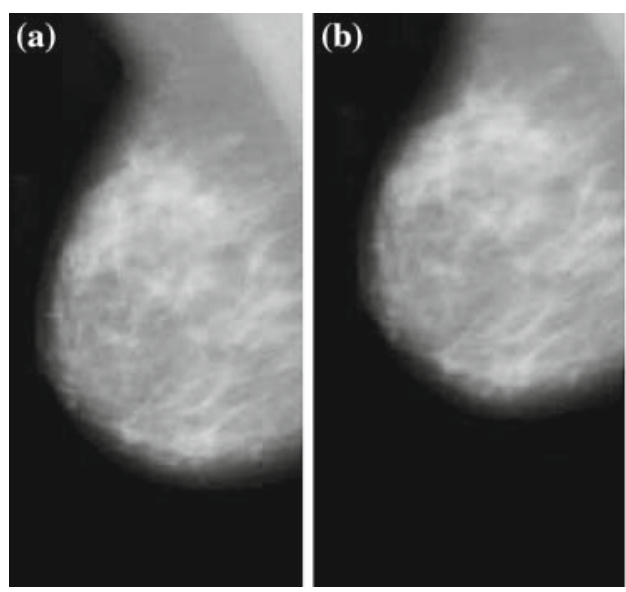

Fig. 4 a The original image used as the floating image, b artificially deformed image used as the reference image, c the registered image using the proposed TPS approximation, d the registered image using Rueckert's approach

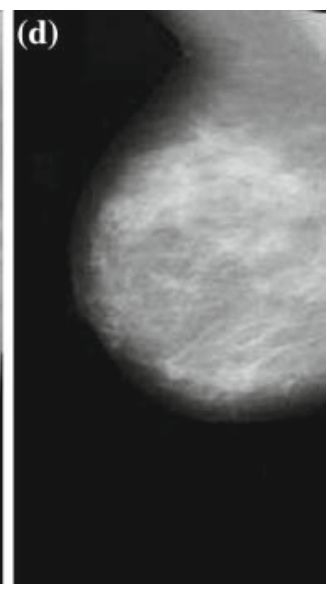

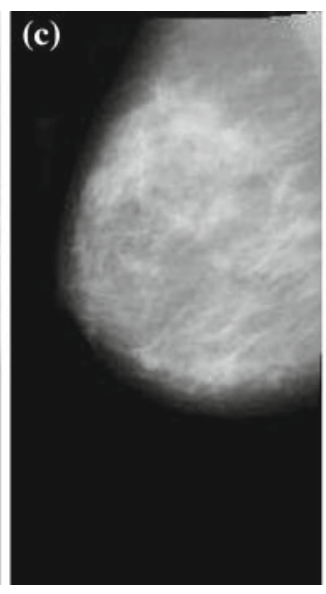


The TPS approximation approach proposed in this publication allows to incorporate anisotropic landmark errors as well as rotational information. The integration of the method into an intensity-based hierarchical non-rigid registration framework is straightforward and improved the registration result significantly. In contrast to the state-of-the-art, our method is fully automatic. The proposed TPS approximation incorporating rotation and landmark errors is well suited for integration in other applications using TPS interpolation. The generalization of the proposed procedure to 3D images is straightforward, as only the definition of the angular landmarks needs to be adjusted.

\section{References}

1. Andronache A, Cattin Ph, Szekely G (2005) Adaptive subdivision for hierarchical non-rigid registration of multi-modal images using mutual information. Med Image Comput Comput Assist Interv 2005( 90):976-983

2. Arsigny V, Pennec X, Ayache N (2003) A novel family of geometrical transformations: polyrigid transformations. Application to the registration of histological slices. INRIA Res Rep 4837:39

3. Berthaud C, Bourennane E, Paindavoine M, Milan C (1998) Implementation of a real time image rotation using b-spline interpolation on fpga's board. Int Conf Image Process 98(3):995-999

4. Bookstein FL (1989) Principal warps: thin-plate splines decomposition of deformation. IEEE Trans Pattern Anal Mach Intell 11:567-585

5. Bookstein FL, Green DK (1993) A feature space for edgels in images with landmarks. J Math Imaging Vis 3:231-261
6. Chen Y, Dass S, Ross A, Jain A (2005) Fingerprint deformation models using minutiae locations and orientations. Appl Comput Vis IEEE Workshop Motion Video Comput 1:150-155

7. Duchon J (1977) Splines minimizing rotation-invariant seminorms in Sobolev spaces. Construct Theory Funct Several Variables 1:85100

8. Hill DLG, Batchelor PG, Holden M, Hawkes DJ (2001) Medical image registration. Phys Med Biol 46:R1-R44

9. Lewis J, Hwang HJ, Neumann U, Enciso R (2004) Smart point landmark distribution for thin-plate splines. SPIE Med Imaging 370:1236-1243

10. Mardia KV, Kent JT (1996) Kriging and splines with derivative information. Biometrika 83:207-221

11. Rohr K (1998) Image registration based on thin-plate splines and local estimates of anisotropic landmark localization uncertainties. In: Proceedings of the first international conference on medical image computing and computer-assisted intervention (MICCAI '98), pp 1174-1183

12. Rohr K, Fornefett M, Stiehl HS (2003) Spline-based elastic image registration: integration of landmark errors and orientation attributes. Comput Vis Image Understand 90:153-168

13. Rohr K, Sprengel R, Stiehl HS (1997) Incorporation of landmark error ellipsoids for image registration based on approximating thinplate splines. In: Proceedings of computer assisted radiology and surgery (CAR '97), pp 234-239

14. Rueckert D, Sonoda LI, Hayes C, Hill DLG, Leach MO, Hawkes DJ (1999) Nonrigid registration using free-form deformations: application to breast MR images. IEEE Trans Med Imaging 18:712-721

15. Schaefer $\mathrm{S}$, McPhail $\mathrm{T}$, Warren $\mathrm{J}$ (2006) Image deformation using moving least squares. ACM Trans Graph 25:533-540

16. Zitova B, Flusser J (2003) Image registration methods: a survey. Image Vis Comput 21(11):977-1000 